\title{
Initiative promotes youth development, healthy living, science literacy
}

\begin{abstract}
$\longrightarrow$ alifornia's youth are in trouble, facing challenges from alarmingly high obesity and school dropout rates to alarmingly low sci-
\end{abstract} ence literacy rankings. While these trends have been easy to spot, reversing them has been difficult. To help identify - and implement - effective solutions to these urgent problems, UC Cooperative Extension (UCCE) is partnering with communities statewide via

\section{One in six youths aged 16 to} 24 years is out of school and out of work.

UCCE Community Development Specialist Dave Campbell, who leads the new youth-focused initiative. "If our work is going to be relevant to the real world, we need to reflect its complexity."

\section{Healthy Families and Communities}

Called Healthy Families and Communities, the initiative includes three interwoven strands: encouraging healthy lifestyles, boosting science literacy, and fostering positive youth development. The need is great. A third of school-aged children in California are overweight or obese, and at current rates nearly half of the state's adults could be obese by 2030, according to

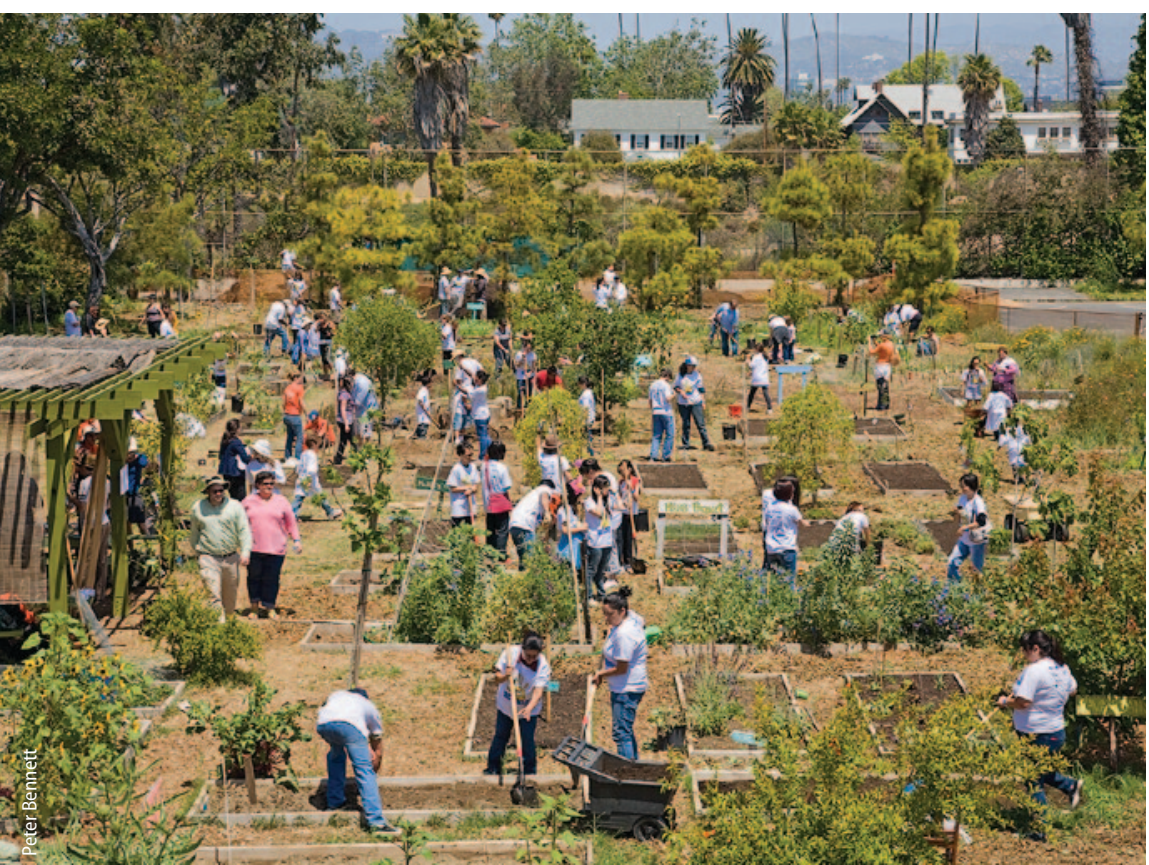

Students, parents and teachers work on the garden at the 24th Street School garden in Los Angeles on Big Sunday, the nation's largest annual citywide community service event. This prototype garden classroom spans more than one acre and includes an orchard with 55 fruit trees. a 2012 report called $F$ as in Fat: How Obesity Threatens America's Future by the nonprofit Trust for America's Health. Obesity is linked to chronic illnesses, including type 2 diabetes, heart disease and high blood pressure. In 2009, the California Center for Public Health Advocacy estimated the cost to the state of overweight, obesity and physical inactivity was more than $\$ 41$ billion per year, the highest nationwide.

California's science literacy is also abysmal, with only the District of Columbia scoring worse in eighth-grade science in the National Assessment of Educational Progress's 2011 State Snapshot Report. A workforce with the knowledge and skills for scientific careers is critical to the state's economy, and understanding science is key to participating fully in today's technological society. "It's very important to be able to reason and think critically," Campbell says. "We need an informed citizenry." Equally dismal are the state's high youth dropout rates. One in six youths aged 16 to 24 years is out of school and out of work. Besides having high social costs, this is a missed opportunity for training skilled workers to replace those close to retirement.

Because the challenges facing California's youth are multifaceted, solving them will require a multipronged approach. "We shouldn't think of these issues as discrete - they're interconnected," Campbell says. He cites the school gardening movement as an example of effectively integrating nutrition, science education and youth development. UCCE teaching and extension programs across California communities are informed by UC ANR's active research efforts. Since 2009, UC ANR academics have published more than 100 peer-reviewed research articles addressing one or more of the Healthy Families and Communities strategic initiative's priorities of healthy living for obesity prevention, youth science literacy and positive youth development (http://ucanr.edu/sites/HFC/).

\section{Shaping Healthy Choices}

"You need to address several components at once to make a change," agrees UCCE Nutrition Science Specialist Sheri Zidenberg-Cherr, who codirects the UC Davis Center for Nutrition in Schools. She leads a multidisciplinary team that, with funding from an ANR competitive grant of $\$ 600,000$ over 4 years, is evaluating a multicomponent K-6 nutrition education effort called the Shaping Healthy Choices Program.

Designed to both improve child health and support local agriculture, the program incorporates serving regional fruits and vegetables, a salad bar, a hands-on garden, and classroom nutrition and physical fitness lessons. Just as importantly, the program is aligned 


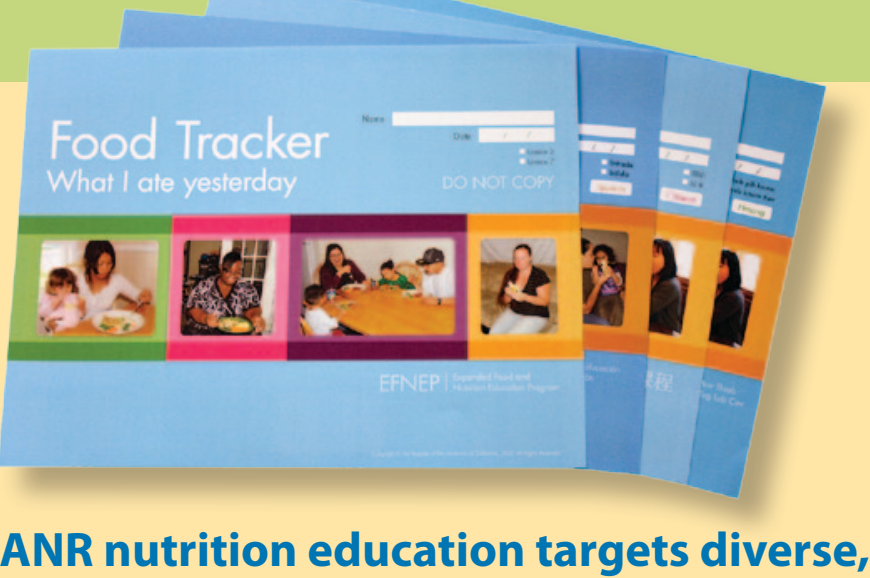 low-literacy communities}

ANR has developed new nutrition education materials tailored to California's low-literacy and culturally diverse communities. These materials are part of the Expanded Food and Nutrition Education Program (EFNEP), a federally funded program that offers nutrition education to limited-resource families and children.

The new materials include pictures and are easy to read. "We reduced the literacy level from the eighth-grade level to the third-grade level," says Connie Schneider, who directs ANR's Youth, Families and Communities program, which administers EFNEP in California. The materials have also been translated into several languages and are tailored to the individual cultures. "Before, we just had English and Spanish — now we also have Chinese, Hmong and Vietnamese," she adds. "We're giving people the information they need to make good choices."

In 2012, the University of California's EFNEP Checklist and Instruction Guide won a merit award from the Health Information Resource Center (HIRC), a national clearinghouse for consumer health information programs and materials. The guide, which includes a food behavior checklist, is multilingual and culturally customized; it is called the Food Behavior Checklist for Low-literate Audiences in English, Spanish, Chinese, Vietnamese and Hmong with Instruction Guide. The HIRC National Health Information Awards program

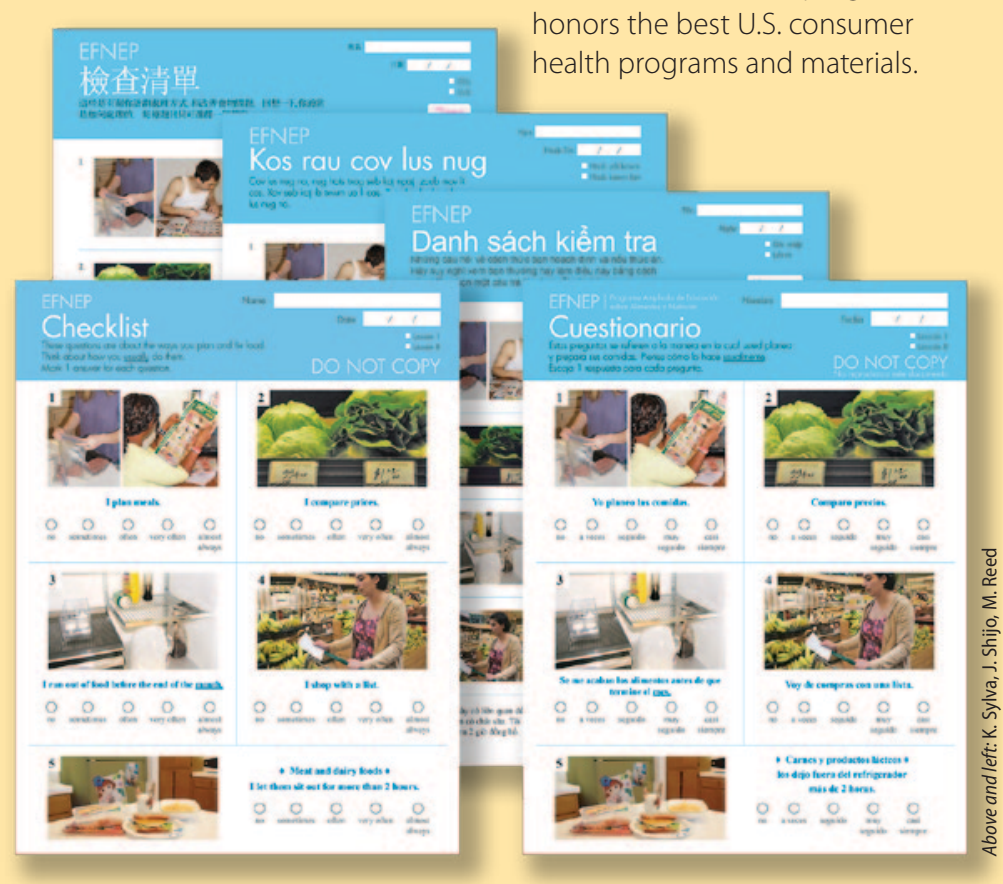

Evaluation tools that are appropriate to a target audience's language, culture and literacy level will increase accuracy of participant data, enabling UC Cooperative Extension educators to better address participants' needs. with the California State Board of Education's core content standards in science, math, language arts and social studies. "This is critical for teacher buy-in," Zidenberg-Cherr says.

The study is controlled, making it a rarity in the field of community research. Investigators will study matched schools in Northern and Central California, comparing those that are implementing the Shaping Healthy Choices Program with those that are not. Controls allow researchers to sort out behavior changes that are due to a given program from those that happen by chance or that are due to other environmental factors. "This is the only way to demonstrate that this type of program actually increases healthy behaviors," Zidenberg-Cherr says. "If the assessment is positive, we can inform policy, and other schools around the state can use our intervention schools as models."

Measures of success include whether children make healthier choices in the lunchroom, actually eat more of the fruits and vegetables they put on their plates and are more likely to try new produce such as jicama and kohlrabi. "We want to open up their minds to trying new foods instead of saying, 'This is all you're going to get'," she says. "We're saying, 'This is really enjoyable, you're growing it with your hands in the dirt, try this!"'

\section{Inquiry-based learning}

Another innovative aspect of the Shaping Healthy Choices Program is the curriculum. In contrast to the old-style workbook-driven lessons, students follow their interests through the curriculum. "They learn to ask questions instead of just listening to a lecture," Zidenberg-Cherr says.

Called inquiry-based learning, this approach also includes application to the real world. For example, after a lesson on food labels at school, students compare food labels on their own at home and in grocery stores. "Application is what makes learning stick," says Martin Smith, an associate UCCE specialist in youth science literacy, who helped develop the program's curriculum. "Inquiry-based learning takes longer, but it's deeper - kids own the knowledge because they figured it out themselves."

Inquiry-based learning also hones critical-thinking skills. After collecting and comparing information from nutrition labels, for example, children can make data-based decisions about what to eat. "It's part of scientific literacy, and hopefully over time they will make better choices," Smith says.

\section{Teaching the teachers}

Smith also instills the basics of inquiry-based learning in 4-H leaders, volunteers who provide nonformal education outside the classroom. He does this with inquiry-based training. "You can't teach it with 


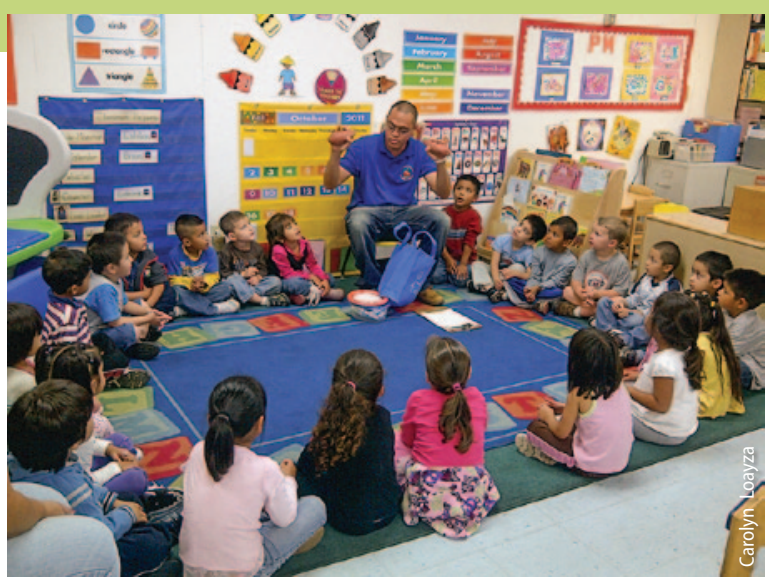

Shaping Healthy Choices incorporates serving regional fruits and vegetables, a hands-on garden, and classroom nutrition lessons. It is aligned with California's core content standards in science, math, language arts and social studies.

lectures or PowerPoints," he says. "They have to experience it themselves, and then they have an 'aha!' moment and say, 'I see what you mean'." The process is iterative, with the 4-H leaders meeting again to share what did and didn't work in their clubs. "They build knowledge and skills based on real-life experience," Smith says. "This makes them much better facilitators of inquiry-based learning. They've been there themselves and know how to encourage students."

Inquiry-based learning is a natural fit with 4-H, which emphasizes a "learn by doing" approach that boosts science literacy by encouraging exploration and critical thinking. Aimed mostly at upper elementary and middle school students, the 4-H curriculum includes animal education units ranging from veterinary science to livestock biosecurity as well as environmental education units ranging from water quality to protecting pollinators. Still in the works are units on animal welfare and preharvest food safety. "We're working toward integrating these systematically to have broader community impacts statewide," Smith says.

\section{Healthy Living}

Another component of $4-\mathrm{H}$ is the Healthy Living initiative, which is a holistic approach to well-being, including physical fitness as well as choosing, growing and cooking healthy foods. As part of the 2013 centennial of 4-H in California, the Revolution of Responsibility Centennial Campaign is partnering with the 4-H Foundation to fund 1,000 servicelearning projects at $\$ 1,000$ each. "Service learning is core to the 4-H experience," says Connie Schneider, who directs Youth, Families and Communities, a statewide UC ANR program that includes nutrition programs such as the Expanded Food and Nutrition Education Program (see sidebar) and youth development programs such as 4-H. "In Healthy Living and other youth-led projects, learning takes place through leadership, collaboration and civic engagement," she adds. "These projects provide opportunities to make a difference in communities."
The Yolo County Cottonwood 4-H Club, for example, was awarded a grant for a school nutrition education project aimed at inspiring healthier eating habits. Implemented at a local elementary school, the project included classroom nutrition lessons, weekly deliveries of fresh fruit for 10 weeks, taste tests of seasonal fruits and making bicycle-powered fruit smoothies. A video of this project has been featured on the web page of the California Health and Human Services Agency's "Let's Get Healthy California" Task Force.

\section{Obesity prevention}

Important as it is to increase consumption of fruits and vegetables, that alone is not enough to combat obesity. "Increasing any kind of food without reducing overall calories won't make a dent in children's BMIs," says UCCE Nutrition Specialist Pat Crawford. BMI, or body mass index, is calculated from a person's weight and height, and is a proxy for measuring body fat. For children, overweight is defined as a BMI between the 85th and 95th percentile for chil-

\section{A third of school-aged children in California are overweight or obese,} and at current rates nearly half of the state's adults could be obese by 2030 . dren of the same age

and sex on pediatric growth charts, while obesity is defined as a BMI in the 95th percentile or above.

"Children are three times heavier than they were 30 years ago. Never have we seen increases of this magnitude," Crawford says. As childhood obesity has risen, so have the accompanying health risks. The number of adolescents with diabetes or prediabetes, for example, has risen from nearly $10 \%$ to nearly $25 \%$ in just the last decade. "How can we as a society say that's OK? These are our children!" she says.

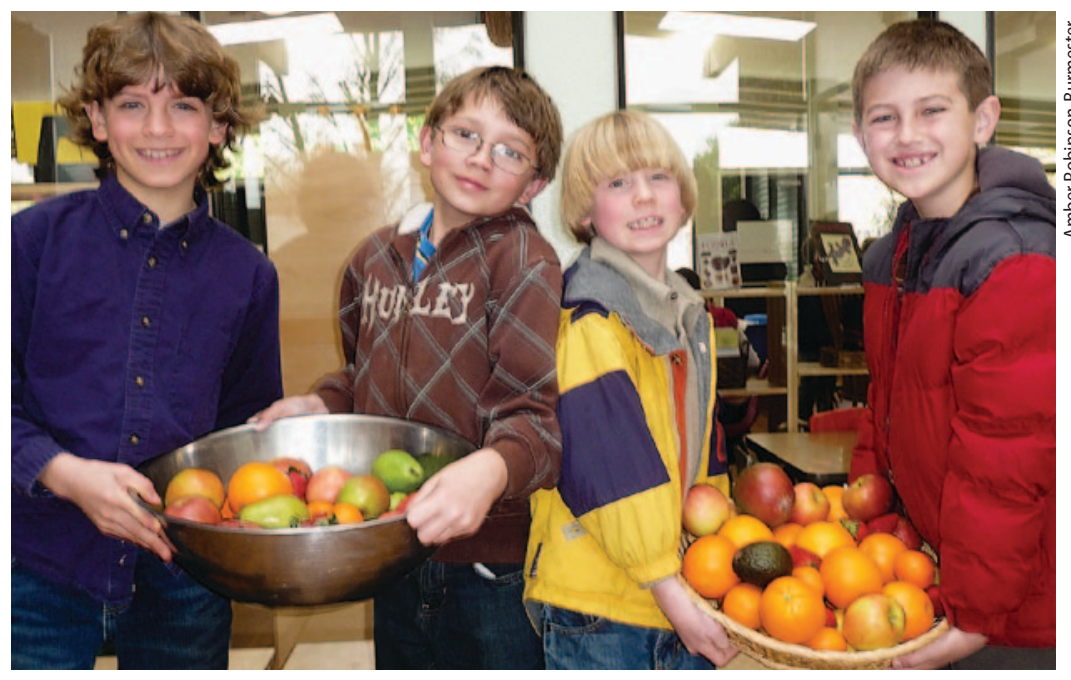

As part of the Cooperative Extension Centennial Campaign, the Yolo County Cottonwood 4-H Club received a grant for a nutrition project to inspire healthier eating habits. It included classroom nutrition lessons, weekly deliveries of fresh fruit for 10 weeks, taste tests of seasonal fruits and making bicycle-powered fruit smoothies. 


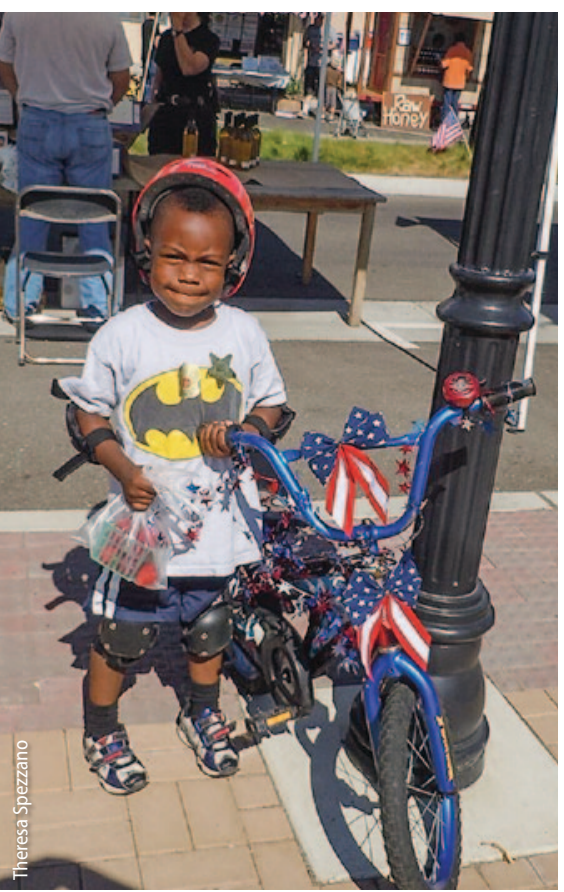

Fresh fruit can substitute for sweetened beverages and fast food, two of the strongest factors driving obesity. Above, bicyclist participates in Get Fit Riverbank, a summer program in Stanislaus County.
Two of the strongest factors driving obesity are sweetened beverages and fast food, and decreasing their consumption is just as important as increasing the consumption of healthy foods. "You have to do both," Crawford says. "In the past, we worked to help people increase their intake of healthy foods, and now we realize that they also need to decrease their intake of less healthy foods."

She leads a multidisciplinary team that, with funding from an ANR competitive grant over the next 2 years, is evaluating a community-based approach to preventing obesity in elementary school children. This work was inspired partly by a 2012 report called Accelerating Progress in Obesity Prevention: Solving the Weight of the Nation from the National Academy of Sciences' Institute of Medicine. "The report cited the importance of a community-based approach to preventing obesity and identified Cooperative Extension as the on-the-ground leaders who could do this," she says. "It was almost a call for action."

The obesity prevention program under evaluation links schools and after-school programs with UCCE county nutrition advisors and $4-\mathrm{H}$. "This is the first attempt by UCCE leaders to link forces in the community in this way to prevent child obesity," she says. "Strong UCCE partnerships in the community are key to our success."

The team is studying fourth- and fifth-grade students who participate in school and after-school obesity prevention programs in Butte and Shasta counties, using BMI reductions after 2 years as a measure of success. "Two-thirds of Americans are overweight or obese, and the old model was to treat them one by one," Crawford says. "The new model is communitywide prevention to keep people from getting obese in the first place and to keep those who are overweight from getting heavier."

While these Healthy Families and Communities efforts underscore the complexity of the challenges facing youth, they also highlight the promise of taking an integrated approach to solving problems from obesity to science illiteracy to youth disengagement. Says Healthy Families and Communities initiative leader Campbell, "If we shift public thinking, policy and resource allocation, we can shift these trends in a positive direction." - Robin Meadows

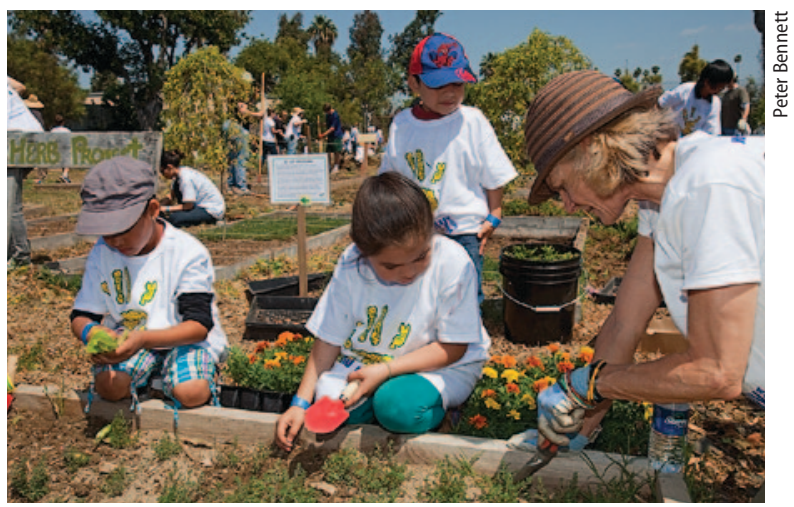

The 24th Street School instructional garden in the West Adams neighborhood of Los Angeles includes California native gardens, 16 vegetable production beds, an orchard of fruit trees, and shaded teaching areas.

\section{For more information}

Atkins Center for Weight and Health

http://cwh.berkeley.edu

California Center for Public Health Advocacy. 2009. The Economic Costs of Overweight, Obesity and Physical Inactivity among California Adults. www.publichealthadvocacy.org/costofobesity.htm

California Health and Human Services Agency, "Let's Get Healthy California” Task Force. www.chhs.ca.gov/Pages/HealthCalTaskforce.aspx

National Academy of Sciences, Institute of Medicine. 2012. Accelerating Progress in Obesity Prevention: Solving the Weight of the Nation. www.iom.edu/Reports/2012/Accelerating-Progress-in-Obesity-Prevention.aspx

National Assessment of Educational Progress (NAEP). 2011. Science 2011 State Snapshot Report: California. http://nces.ed.gov/nationsreportcard/pdf/stt2011/2012467CA8.pdf

Trust for America's Health. 2012. F as in Fat: How Obesity Threatens America's Future 2012.

http://healthyamericans.org/report/100/

UC ANR Healthy Families and Communities Strategic Initiative. http://ucanr.org/sites/HFC/

Bibliography of UC ANR research on the Healthy Families and Communities Strategic Initiative priorities. http://ucanr.edu/u.cfm?id=55

UC 4-H Youth Development Program www.ca4h.org/ 